\title{
Intracellular survival of Salmonella enterica serovar Typhi in human macrophages is independent of Salmonella pathogenicity island (SPI)-2 \\ Correspondence \\ France Daigle \\ france.daigle@umontreal.ca \\ Received 27 May 2010 \\ Revised 5 August 2010 \\ Accepted 27 August 2010

\author{
Chantal G. Forest,† Elyse Ferraro,† Sébastien C. Sabbagh \\ and France Daigle
} \\ Department of Microbiology and Immunology, University of Montreal, C.P. 6128 Succursale Centre- Ville, Montreal, OC H3C 3J7, Canada

\begin{abstract}
For successful infection, Salmonella enterica secretes and injects effector proteins into host cells (SPIs)-1 and -2. The SPI-2 T3SS is involved in intracellular survival of $S$. enterica serovar Typhimurium and systemic disease. As little is known regarding the function of the SPI-2 T3SS from S. enterica serovar Typhi, the aetiological agent of typhoid fever, we investigated its role for survival in human macrophages. Mutations in the translocon (sseB), basal secretion apparatus $(s s a R)$ and regulator $(s s i B)$ did not result in any reduction in survival under many of the conditions tested. Similar results were obtained with another $S$. Typhi strain or by using human primary cells. Results were corroborated based on complete deletion of the SPI-2 T3SS. Surprisingly, the data suggest that the SPI-2 T3SS of S. Typhi is not required for survival in human macrophages.
\end{abstract} by two distinct type three secretion systems (T3SSs) located on Salmonella pathogenicity islands
}

\section{INTRODUCTION}

Salmonella enterica is an important pathogen of animals and humans, which typically become infected by ingestion of contaminated food or water. There are two major groups of S. enterica that cause disease in humans: the systemic (typhoid fever) group and the non-typhoidal salmonellae group. Typhoid fever is mainly caused by S. enterica serovar Typhi (S. Typhi), a host-adapted serovar that is specific for humans. Non-typhoidal salmonellae are generally associated with gastroenteritis, which rarely develops into an invasive infection. S. enterica serovar Typhimurium is frequently associated with gastroenteritis in humans, whereas this serovar causes a systemic typhoid-like disease in susceptible mice. S. Typhimurium infection in susceptible mice is usually used as a model for $S$. Typhi infection in humans, as these serovars share many virulence factors, including Salmonella pathogenicity islands (SPIs) (McClelland et al., 2001; Parkhill et al., 2001; Sabbagh et al., 2010).

$S$. Typhimurium encodes two distinct type three secretion systems (T3SSs), located on SPI-1 and SPI-2. The SPI-1

†These authors contributed equally to this work.

Abbreviations: $\mathrm{Cl}$, competitive index; IFN- $\gamma$, gamma interferon; MDMs, monocyte-derived macrophages; PBMCs, peripheral blood mononuclear cells; SPI, Salmonella pathogenicity island; T3SS, type three secretion system.

Two supplementary figures, showing the SPI-2 T3SS and the mutants generated during this study, are available with the online version of this paper.
T3SS is involved in invasion of epithelial cells (Galán \& Curtiss, 1989), whereas the SPI-2 T3SS is essential for survival and replication in host cells (Ochman et al., 1996; Shea et al., 1996). The SPI-2 T3SS corresponds to a $25 \mathrm{~kb}$ fragment organized in four operons: regulatory, structural I, structural II and effector/chaperone (see Supplementary Fig. S1, available with the online version of this paper, and Kuhle \& Hensel, 2004). The two-component regulatory system $\mathrm{SsrA} / \mathrm{SsrB}$ is responsible for the transcriptional regulation of SPI-2 operons as well as the regulation of effector genes located outside SPI-2 (reviewed by Haraga et al., 2008). The T3SS needle complex comprises basal component proteins in the inner membrane (including SsaR), proto-channel proteins, outer membrane proteins, proteins forming a hollow tube generating the needle and outer ring proteins (including SseB), which form the translocon that traverses the host vacuolar membrane (Supplementary Fig. S1). This SPI-2 T3SS injects at least 20 effector proteins across the vacuolar membrane into the host cytosol (Haraga et al., 2008). These effectors interact with the host machinery and are required for modification of the vacuole and modulation of intracellular processes (Brumell et al., 2001; Waterman \& Holden, 2003; Yu et al., 2004), which will allow the bacteria to survive and replicate in a membrane-bound structure called the Salmonella-containing vacuole. Intracellular induction of SPI-2 genes was initially observed by using reporter assays (Valdivia \& Falkow, 1997; Cirillo et al., 1998; Pfeifer et al., 1999) and was confirmed by microscopy (Kuhle \& Hensel, 2002), microarray data (Eriksson et al., 2003) and Western blotting (Coombes et al., 2007). 
Survival and replication of $S$. Typhimurium within cultured cells correlate with systemic survival and persistence in animal models, as mutants that fail to replicate in cultured cells are avirulent in animals (Fields et al., 1986; Leung \& Finlay, 1991; Shea et al., 1999). Survival within macrophages is therefore an essential step for Salmonella pathogenesis. Two major systems are implicated in this process: (1) the two-component regulatory system PhoPQ and its regulon (Groisman et al., 1989; Miller et al., 1989; Garvis et al., 2001) and (2) the SPI-2 T3SS (Shea et al., 1996; Cirillo et al., 1998; Hensel et al., 1998). The intracellular proliferation defect of SPI-2 mutants was observed in primary macrophages and several macrophagelike cell lines as well as in epithelial cell lines (Ochman et al., 1996; Cirillo et al., 1998; Hensel et al., 1998; Beuzón et al., 2002). S. Typhimurium strains deficient in SPI-2 are highly attenuated in the murine systemic infection model (Shea et al., 1996; Hensel et al., 1998; Chan et al., 2005).

The intracellular survival defect can be observed in most individual mutants, such as $s s e B, s s a R$ and $s s r B$. The $s s e B$ mutant of $S$. Typhimurium has a growth defect in murine macrophages (peritoneal, RAW264.7 and J774A.1), and in epithelial cells (HEp-2) but was not recovered from liver or spleen of infected $\mathrm{BALB} / \mathrm{c}$ mice (Cirillo et al., 1998; Hensel et al., 1998). SseB is a translocon constituent involved in pore formation in the host membrane for delivery of effector proteins. An sseB mutant does not secrete SseD and SseC, two other translocon proteins (Chakravortty et al., 2005), and SseB is required for the translocation of effector proteins by intracellular Salmonella (Nikolaus et al., 2001). Also, ssaR mutants of $S$. Typhimurium are defective in intracellular replication in murine macrophages (RAW264.7), are attenuated in BALB/c mice and are unable to secrete SPI2 effectors (Shea et al., 1996; Coombes et al., 2004). Mutants of the two-component system SsrAB are unable to survive within murine macrophages (RAW264.7, J774A.1), are impaired in epithelial cell replication (HEp-2) and are avirulent in mice (Cirillo et al., 1998; Hensel et al., 1998; Ochman et al., 1996; Shea et al., 1996).

Moreover, SPI-2 has also been shown to interfere with antigen presentation (Cheminay et al., 2005; Tobar et al., 2006; Halici et al., 2008), cytokine production (Uchiya et al., 2004) and cytotoxicity (Monack et al., 2001; Paesold et al., 2002). The critical role of the SPI-2 T3SS for S. Typhimurium is therefore well established. Although most SPI-2 genes were upregulated following $S$. Typhi uptake by human macrophages (Faucher et al., 2006), the specific role of the SPI-2 T3SS of S. Typhi has not yet been reported. In this study, we targeted specific components of the secretion system, such as the translocon $(\operatorname{sse} B)$, the basal secretion apparatus $(s s a R)$ and the regulator $(s s r B)$ in order to investigate the contribution of the SPI-2 T3SS during interaction of $S$. Typhi with human macrophages.

\section{METHODS}

Bacterial strains and growth conditions. Strains and plasmids used in this study are listed in Table 1. Bacteria were routinely grown overnight statically (low aeration) in Luria-Bertani (LB) broth, unless indicated otherwise. When required, antibiotics or supplements were added at the following concentrations: $50 \mu \mathrm{g}$ ampicillin $\mathrm{ml}^{-1}, 34 \mu \mathrm{g}$ chloramphenicol ml $\mathrm{m}^{-1}, 50 \mu \mathrm{g}$ nalidixic acid $\mathrm{ml}^{-1}$ or $50 \mu \mathrm{g}$ diaminopimelic acid $\mathrm{ml}^{-1}$.

Construction of SPI-2 mutants. Deletion of $s s e B$, ssaR and the entire T3SS of SPI-2 $[\Delta(s s a U-s s r B)]$ were generated by allelic exchange as described previously for the $s s r B$ deletion (Faucher et al., 2009) by using the overlap-extension PCR method (Basso et al., 2002). Primers are listed in Table 2, and detailed information on the deletions is presented in Supplementary Fig. S2. Mutations were confirmed by PCR. All mutants have growth curves in LB broth similar to that of the wild-type strain (data not shown).

SseB antibodies and secretion assays. The amplified $s s e B$ gene of $S$. Typhi ISP1820 was cloned into pET-21a vector (pSIF128) and SseB proteins were purified by using Ni-nitrilotriacetic acid agarose beads (Qiagen) and polyclonal antibodies against SseB were raised in rabbits (GeneScript). For secretion assays, bacteria were grown in a low phosphate and magnesium medium (LPM; $\mathrm{pH} 5.8$ ), corresponding to SPI-2-inducing conditions (Coombes et al., 2004). Bacteria were pelleted, and supernatant was collected and precipitated as described previously (Faucher et al., 2009). Samples were dissolved in an appropriate volume of SDS-PAGE loading buffer, according to the $\mathrm{OD}_{600}$ value of the original culture. Proteins were separated on 10 or $15 \%(w / v)$ SDS-polyacrylamide gels and then transferred onto PVDF membranes and incubated with rabbit anti-GroEL (Sigma) or rabbit affinity-purified antibodies raised against recombinant SseB. Peroxidase-conjugated AffiniPure goat anti-rabbit IgG (Jackson ImmunoResearch Laboratories) was used as the secondary antibody and was detected with the ECL plus Western blotting detection reagent (GE Healthcare).

Infection of human cultured macrophages. The human monocyte cell line THP-1 (ATCC TIB-202) was maintained in RPMI 1640 (Wisent) containing $10 \%(\mathrm{v} / \mathrm{v})$ heat-inactivated fetal calf serum (Invitrogen), $25 \mathrm{mM}$ HEPES (Wisent), $2 \mathrm{mM}$ L-glutamine, $1 \mathrm{mM}$ sodium pyruvate and $1 \%$ modified Eagle's medium with nonessential amino acids (Wisent). A stock culture of these cells was maintained as monocyte-like, non-adherent cells at $37^{\circ} \mathrm{C}$ in an atmosphere containing $5 \%(\mathrm{v} / \mathrm{v}) \mathrm{CO}_{2}$. For macrophage infection, cells were seeded at $5 \times 10^{5}$ cells per well in 24-well tissue-culture dishes and were differentiated by addition of $10^{-7} \mathrm{M}$ phorbol 12myristate 13-acetate for $48 \mathrm{~h}$. Macrophage infection was performed as described previously (Daigle et al., 2001) unless specified otherwise. Briefly, bacteria were grown overnight without shaking in LB broth, usually reaching an $\mathrm{OD}_{600}$ of 0.6 . Bacteria were added to the cell monolayer at an m.o.i. of $10: 1$, and were centrifuged for $5 \mathrm{~min}$ at $800 \mathrm{~g}$ to synchronize bacterial uptake. After incubation for $20 \mathrm{~min}$ at $37^{\circ} \mathrm{C}$, extracellular bacteria were removed by washing cells three times with prewarmed PBS, pH 7.4, and the infected monolayers were either lysed $\left(\mathrm{T}_{0}\right)$ or incubated for $2 \mathrm{~h}$ with medium containing $100 \mu \mathrm{g}$ gentamicin $\mathrm{ml}^{-1}$ (Wisent) to kill extracellular bacteria, and then with $12 \mu \mathrm{g}$ gentamicin $\mathrm{ml}^{-1}$ for the remainder of the experiment. Cells were lysed by addition of $1 \mathrm{ml} 0.1 \%(\mathrm{w} / \mathrm{v})$ sodium deoxycholate in PBS per well, and the number of surviving bacteria was determined as c.f.u. by plating on LB agar. Survival (fold increase) was defined as the number of bacteria recovered $24 \mathrm{~h}$ after infection divided by the number of bacteria detected $2 \mathrm{~h}$ after infection. Results are expressed as the mean \pm SEM of replicate experiments. The non-parametric Mann-Whitney test was used for statistical analysis. Where indicated, bacteria grown to stationary phase in LB broth were diluted in PBS or 
Table 1. Bacterial strains and plasmids used in this study

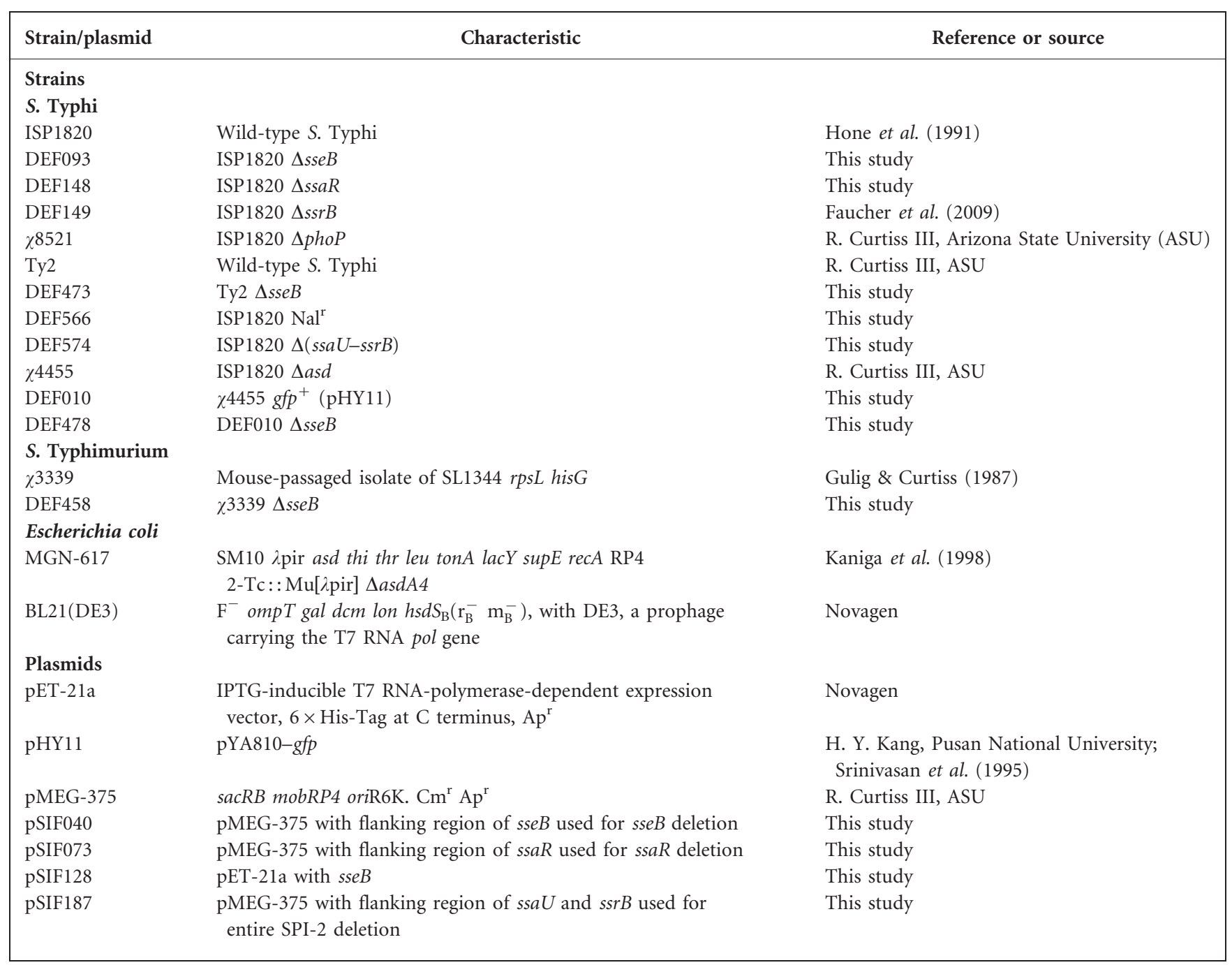

Table 2. Primers used in this study

\begin{tabular}{|ll|}
\hline Primer & \multicolumn{1}{c|}{ Sequence $\left(\mathbf{5}^{\prime} \mathbf{- 3}^{\prime}\right)^{*}$} \\
\hline sseB-BF & CGGGATCCAATACGCACCACGGCTTTT \\
sseB-BR & CCCCGGTGGACCCTACAACAGGCACATGACAC \\
sseB-EF & TTGTAGGGTCCACCGGGGCTTGAGCATTAAG \\
sseB-ER & GCTCTAGAAATGGGACAGGCTCTTAGTTAGCAC \\
ssaR-BF & CGGGATCCTGCTGAGCGTCAACAGATTTATCC \\
ssaR-BR & ATTCGTAACGTCCCCCGTTTTAAGTTGTCG \\
ssaR-EF & ACGGGGGACGTTACGAATTGGAGAGCATGGTTG \\
ssaR-ER & GCTCTAGAAGCAGAAACCCCGCCATATC \\
sseBF-Nde & GGAATTCCATATGTCTTCAGGAAACATCTTATG \\
sseBR-XhoI & CCGCTCGAGTGAATACGTTTTCTGCGCTATCA \\
ssaU-R & CGGGATCCAATACGCTATCTGGTGCTTG \\
ssaU-Fover & TGTTTCGACTGCAGCCTTGTTACGTATGG \\
ssrB-Fover & AAGGCTGCAGTCGAAACACATCGGATGAAT \\
ssrB-R & GCTCTAGAAGGCGTAAGGCTCATCAAAAT \\
\hline
\end{tabular}

${ }^{\star}$ Restriction enzyme sites are underlined. Letters in italics denote overlapping sequences. 
opsonized for $20 \mathrm{~min}$ in $10 \%$ normal human serum before being added to macrophages at an m.o.i of $10: 1$. Activation of macrophages was achieved by addition of $100 \mathrm{U}$ gamma interferon (IFN- $\gamma$ ) $\mathrm{ml}^{-1}$ (PBL Biomedical Laboratories) in the medium $48 \mathrm{~h}$ prior to infection. The addition of IFN- $\gamma$ was maintained throughout the infection.

For competitive index (CI) experiments in macrophages (Segura et al., 2004), a spontaneous nalidixic acid-resistant $\left(\mathrm{Nal}^{\mathrm{r}}\right)$ S. Typhi ISP1820 strain was used as the wild-type strain (DEF566). This Nal ${ }^{\mathrm{r}}$ strain showed no intracellular attenuation compared with strain ISP1820 when both were used in a CI experiment in THP-1 macrophages (data not shown). The wild-type and mutant strains used for competition experiments were separately grown overnight (static) in LB broth, and a 1:1 mixture of the two cultures was added at an m.o.i. of 50:1 to the THP-1 cell monolayer, as described above. The numbers of viable intracellular bacteria were determined 2 and $24 \mathrm{~h}$ after infection by plating on LB agar and on LB agar with antibiotic. The CI for bacterial survival is defined as the mutant to wild-type ratio of bacteria recovered $24 \mathrm{~h}$ post-infection divided by the equivalent ratio of bacteria recovered $2 \mathrm{~h}$ post-infection. Statistical significance was evaluated by using Student's two-tailed $t$ test by comparing ratios (c.f.u. mutant/c.f.u. wild-type) at 24 and $2 \mathrm{~h}$ (Santiviago et al., 2009).

Infection of human monocyte-derived macrophages (MDMs). MDMs were obtained by using previously published protocols (Baron-Bodo et al., 2005; Romieu-Mourez et al., 2006; Goubau et al., 2009). Briefly, peripheral blood mononuclear cells (PBMCs) were isolated by Ficoll gradient centrifugation from fresh apheresis with informed and institutionally approved consent forms from healthy donors. PBMCs were resuspended at $10^{6}$ cells $\mathrm{ml}^{-1}$ in ISCOVE medium (Wisent) supplemented with $2 \%$ human serum (Wisent), antibiotics and $700 \mathrm{U} \mathrm{ml}^{-1}$ recombinant human GM-CSF (a generous gift from Cangene Corporation). On day 7, adherent MDMs were harvested after three washes with PBS and gentle cell scraping and were identified based on assessment of CD14, CD64 and HLA-DR. MDMs were transferred to a 24 -well tissue culture plate and further incubated for 3 days (to day 10). Infection of MDMs with $S$. Typhi was performed as described for THP-1 macrophages.

Microscopy of infected macrophages. THP-1 cells were seeded on to glass coverslips ( $12 \mathrm{~mm}$ diameter) in 24 -well plates. The infection was then carried out using GFP-expressing strains. For fluorescence microscopy, cell monolayers were washed in PBS and then fixed in $4 \%$ paraformaldehyde in PBS, $\mathrm{pH} 7.4$, for $10 \mathrm{~min}$ at room temperature. Coverslips were washed three times in PBS and mounted on Geltol (Immunon Thermo). Samples were analysed using a fluorescence microscope (Eclipse E600; Nikon).

\section{RESULTS}

\section{SPI-2 mutants}

S. Typhi ISP1820 isogenic strains harbouring mutations in genes encoding distinct components of the SPI-2 T3SS were constructed by allelic exchange (Supplementary Figs S1 and S2). Deletion of $s s e B$, encoding a secreted protein, was selected to investigate the effect of a mutation in the translocon. Deletion of $s s a R$ was used to target the basal apparatus component of the needle complex and deletion of $s s r B$ was used to evaluate the effect of a mutation in the regulatory system of the SPI-2 T3SS. To confirm that the SPI-2 mutants (ssaR and $s s r B$ ) of $S$. Typhi were nonfunctional, detection of the SseB protein in the wild-type strain and SPI-2 mutants was monitored by using SPI-2inducing conditions and anti-SseB antibodies. As expected, SseB was secreted in the culture supernatant of the wildtype strain, but not in the supernatant of the SPI-2 mutants (Fig. 1). In the bacterial pellet, the SseB protein was only detected in the wild-type and the $s s a R$ mutant (Fig. 1). The $s s e B$ mutant was used as a control for the specificity of the antibody and GroEL was used as a control for bacterial cytoplasm leakage and was not detected in the culture supernatant. The data confirmed that the SPI-2 mutants were non-functional for secretion.

\section{Phenotype of SPI-2 mutants within human macrophages}

To evaluate bacterial survival within macrophages, THP-1 macrophages were infected with the wild-type strain and the isogenic SPI-2 mutants were grown at low aeration by using a gentamicin protection assay. The number of bacteria present at different times was determined by c.f.u. counts. Surprisingly, none of the specific deletion mutations in SPI-2-T3SS-encoding genes affected bacterial uptake or survival, as the number of bacteria recovered at different times after infection was similar to that of the wild-type strain (Fig. 2). As there were no differences observed between the different SPI-2 mutants, the $s s e B$ mutant was selected for further experiments.

The role of S. Typhi SPI-2 in survival in human macrophages was then assessed more specifically at different times after infection. $S$. Typhi had already begun to replicate $2 \mathrm{~h}$ after infection and continued for up to 3 days after infection (Fig. 3). A growth pattern similar to that for the wild-type strain was observed for the sseB mutant (Fig. 3). S. Typhi constitutively expressing green fluorescent protein (DEF010) and its sseB isogenic mutant (DEF478) were used to visualize bacteria within macrophages by fluorescence microscopy. Initially, many macrophages were infected with a single bacterium or a few bacteria (Fig. 3b). At $24 \mathrm{~h}$ post-infection, infected macrophages contained many bacterial clusters, suggesting that the bacteria had replicated (Fig. 3b). Interestingly, the

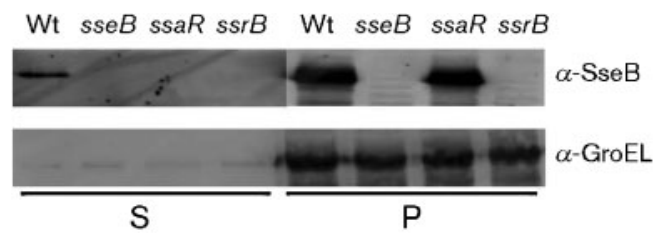

Fig. 1. In vitro secretion of SseB in SPI-2 mutants. Wild-type $S$. Typhi ISP1820 strain (Wt), and isogenic $s s e B, s s a R$ and $s s r B$ deletion mutant strains were grown under SPI-2-inducing conditions (LPM; $\mathrm{pH}$ 5.8). Proteins in the supernatant (S) and bacterial pellet $(P)$ were harvested and examined by Western blotting to detect SseB and GroEL. 


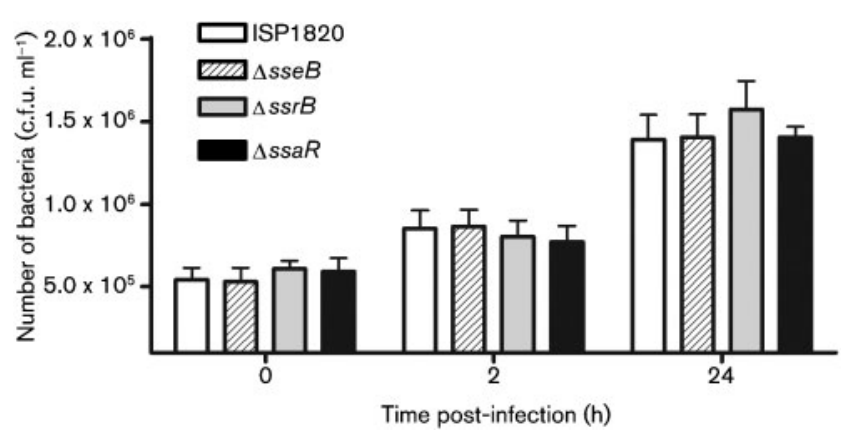

Fig. 2. Effect of mutations in SPI-2 during interactions with human macrophages. THP-1 macrophages were infected with wild-type (ISP1820), sseB, ssaR or ssrB deletion mutant strains and the number of intracellular bacteria were determined at different times after infection. Data presented are the mean \pm SEM of at least three independent experiments performed in duplicate.

infected cell monolayer was mainly intact, even $96 \mathrm{~h}$ postinfection (Fig. 3b) and only a few cells were detached.

To evaluate the usefulness of our infection model, an isogenic strain carrying a phoP deletion, a global transcriptional activator involved in Salmonella virulence and intracellular survival within macrophages (Miller et al., 1989), was used as a control. The isogenic phoP mutant showed significantly reduced survival (Fig. 4), suggesting that the THP-1 macrophages are efficient for bacterial killing.

We next investigated if the absence of survival defects for an SPI-2 mutant was unique to the $S$. Typhi strain ISP1820. An $s s e B$ deletion was generated in $S$. Typhi strain Ty2 and no significant difference in intracellular survival between the wild-type strain and the SPI-2 mutant was observed (Fig. 4). As a positive control, the role of SPI-2 of S. Typhimurium in human macrophages was then investigated. An sseB mutant of S. Typhimurium strain SL1344 was constructed and its contribution to survival in macrophages was evaluated. The $s s e B$ deletion in $S$. Typhimurium significantly reduced intracellular survival of the SPI-2 mutant in THP-1 macrophages (Fig. 4).

\section{Influence of growth conditions}

Salmonella can enter macrophages by several endocytic processes, which can be affected by the bacterial growth conditions prior to infection. Bacteria were grown to late exponential phase under low aeration (static), and were grown to stationary phase with or without opsonization. Regardless of the growth conditions prior to infection, there was no difference between the wild-type and the sseB mutant (Fig. 5). The uptake level of bacteria grown in different conditions was also similar. The number of bacteria recovered after $24 \mathrm{~h}$ of infection was always higher than the number of bacteria recovered after $2 \mathrm{~h}$ (fold increase $>1$ ) when bacteria were grown to late exponential phase without agitation (Figs 2-5). However, when $S$. Typhi uptake was investigated by using bacteria grown with shaking to stationary phase, with or without opsonization, a constant number of bacteria between 2 and $24 \mathrm{~h}$ after infection (fold increase of $\cong 1$ ) was observed (Fig. 5).

\section{Activation of macrophages}

IFN- $\gamma$ is a critical cytokine that plays a central role in host defence against intracellular pathogens, including Salmonella

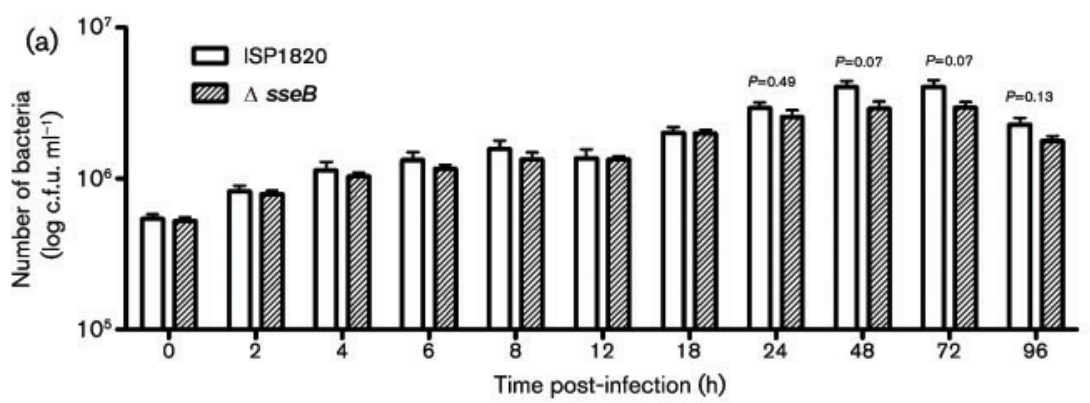

(b)
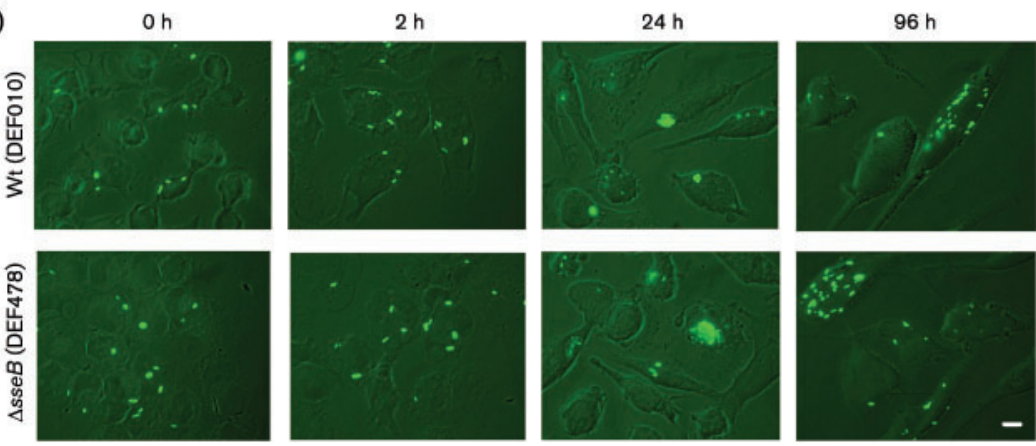

Fig. 3. Time course of $S$. Typhi survival in human macrophages. THP-1 macrophages were infected with S. Typhi ISP1820 and its isogenic sse $B$ mutant. (a) The number of intracellular bacteria were determined at different times after infection. Data presented are the mean \pm SEM of three independent experiments performed in duplicate. $P$-values are indicated for late time points. (b) Fluorescence microscopy of fixed macrophages at different times after infection with bacteria constitutively expressing the green fluorescent protein (original magnification, $\times 1000$ ). 


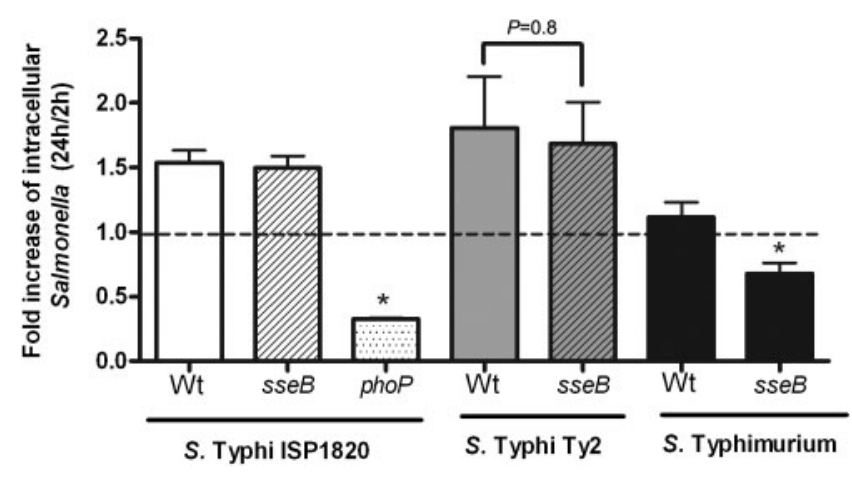

Fig. 4. Role of SPI-2 in intracellular survival in different strains of Salmonella. THP-1 macrophages were infected with $S$. Typhi ISP1820 (Wt) and its isogenic sseB and phoP mutants, S. Typhi strain Ty2 and its isogenic sse $B$ mutant, and $S$. Typhimurium SL1344 and its isogenic sseB mutant. Intracellular survival was determined by comparing the number of bacteria $24 \mathrm{~h}$ postinfection with the number of bacteria at $2 \mathrm{~h}$ (fold increase). Data presented are the mean \pm SEM of at least three independent experiments performed in duplicate. Mutants demonstrating a significant $(P<0.05)$ difference compared with the isogenic wildtype strain are indicated $\left(^{*}\right)$.

(Muotiala \& Mäkelä, 1993; Rosenberg et al., 2002). As Salmonella may encounter activated macrophages during its life cycle inside the host, we investigated the role of SPI-2 during infection of activated macrophages. THP-1 macrophages were primed with IFN- $\gamma$ for $48 \mathrm{~h}$ and then infected

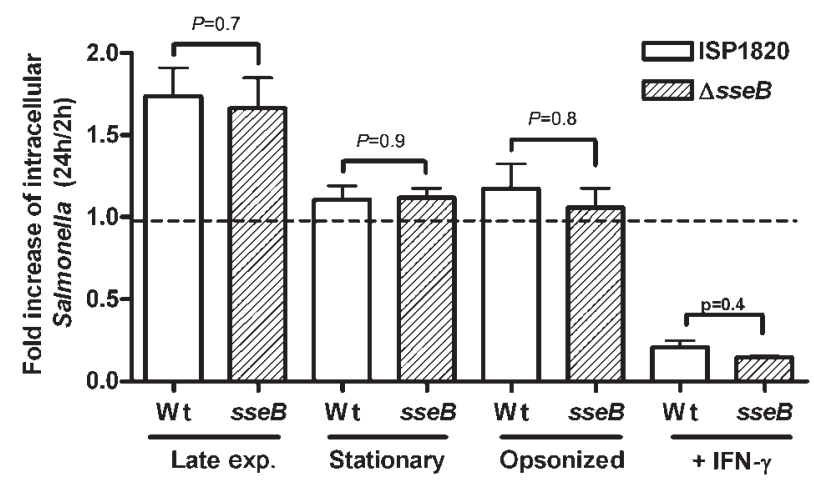

Fig. 5. Effect of growth conditions, opsonization or macrophage activation on intracellular survival of $S$. Typhi. THP-1 macrophages were infected with S. Typhi ISP1820 (Wt) and its isogenic sseB mutant grown in LB: cultures were overnight static (late exponential growth), overnight shaking (stationary phase) with or without opsonization with human serum (opsonized), or by using activated macrophages primed with IFN- $\gamma$. Intracellular survival was determined by comparing the number of bacteria $24 \mathrm{~h}$ postinfection with the number of bacteria at $2 \mathrm{~h}$ (fold increase). Data presented are the mean $\triangle$ SEM of at least three independent experiments performed in duplicate. Exact $P$-values are also indicated. with wild-type $S$. Typhi and its isogenic sseB SPI-2 mutant. Following IFN- $\gamma$ treatment, a small number of bacteria were recovered from macrophages (Fig. 5), although there was no significant difference between the wild-type and the SPI-2 mutant in the IFN- $\gamma$-primed macrophages.

\section{Mixed infection assay of human macrophages}

Survival of the wild-type strain and its isogenic mutants was evaluated simultaneously by using the CI assay. The CIs for the sseB and phoP mutants within THP-1 cells after mixed infections with the wild-type strain were 1.19 $(P=0.50)$ and $0.27(P=0.0048)$, respectively. These results are in agreement with our data from individual strains during infection and confirm that SPI-2 does not affect survival in human macrophages and that the PhoPQ regulon is of importance.

\section{Infection of human MDMs}

It has been shown that macrophages from immortalized cell lines allow moderate growth of an SPI-2 mutant of $S$. Typhimurium compared with a greater level of growth defect in primary cells (Hensel et al., 1998; Helaine et al., 2010). Human MDMs were infected with the wild-type $S$. Typhi strain and the isogenic sseB mutant. The number of bacteria recovered decreased over time, suggesting a defect in survival; however, the number of recovered bacteria was similar for both the wild-type strain and the SPI-2 isogenic mutant (Fig. 6).

\section{Complete deletion of the SPI-2 T3SS}

To validate and understand the surprising results obtained with the different SPI-2 mutants, a $25 \mathrm{~kb}$ deletion of the entire T3SS (ssaU-ssrB) was generated and tested for survival in THP-1 macrophages. Deletion of the T3SS of SPI-2 did not affect bacterial uptake or survival, as the

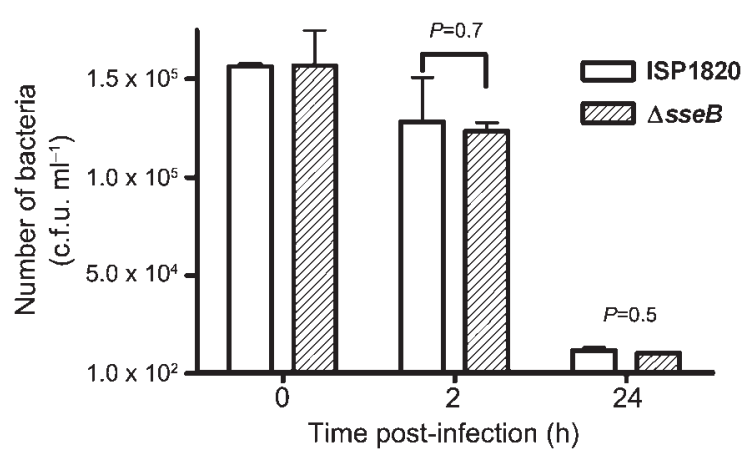

Fig. 6. Role of $S$. Typhi SPI-2 in human MDMs. Cells were infected with S. Typhi ISP1820 and its isogenic sseB mutant and intracellular bacteria were determined at different times after infection. Representative mean \pm SEM of a duplicate MDM experiment from a single donor are shown. $P$-values are indicated. 
number of bacteria recovered at different times after infection was similar to that of the wild-type strain (Fig. 7).

\section{DISCUSSION}

As the ability to survive and replicate inside macrophages is tightly associated with the $S$. Typhimurium SPI-2 T3SS and with virulence (Fields et al., 1986), and since very little information regarding the involvement of S. Typhi SPI-2 T3SS in this process is available, the role of the $S$. Typhi SPI-2 T3SS inside human macrophages was investigated. Mutants corresponding to specific components of the secretion system were tested for intracellular survival, including a translocon mutant ( $s s e B$ ), an apparatus mutant $(s s a R)$ and a transcriptional regulator mutant $(s s r B)$, the last of these being required for expression of SPI-2 genes. These SPI-2 mutants were previously shown to be defective in intracellular replication of $S$. Typhimurium (Cirillo et al., 1998; Hensel et al., 1998). Moreover, any mutations in SseB lead to structural alterations that abolish translocon function, resulting in replication defects in macrophages (Hölzer \& Hensel, 2010). SseB was not secreted in the $s s a R$ and $s s r B$ mutants, confirming that the SPI-2 T3SS is not functional (Fig. 1). Surprisingly, mutations in either $s s e B$ (translocon), ssaR (basal component) or $s s r B$ (regulator), all of which abrogate SPI-2 T3SS function or expression, did not affect $S$. Typhi uptake and survival in human cells. Similar results were obtained during competition assays, when the entire SPI-2 T3SS $(\Delta s s a U-s s r B)$ was deleted (Fig. 7), or when macrophages derived from human monocytic cells were used (Fig. 6), confirming that intracellular survival of $S$. Typhi inside human macrophages is independent of the SPI-2 T3SS. The number of intracellular bacteria corresponding to the sseB SPI-2 mutant increased inside macrophages with a pattern

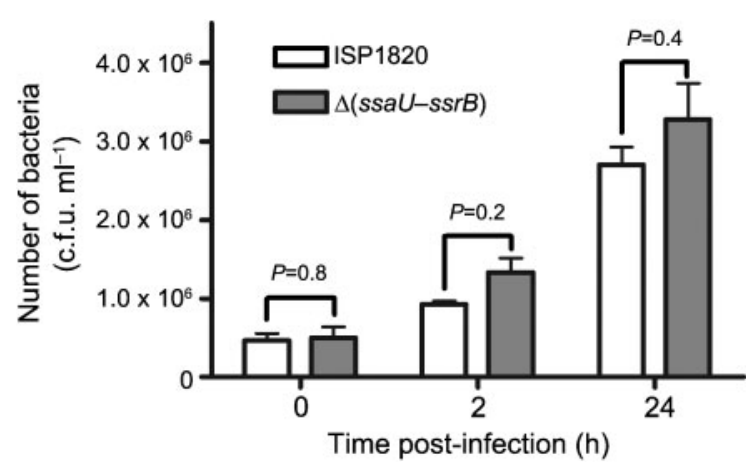

Fig. 7. Effect of complete deletion of the SPI-2 T3SS locus in S. Typhi during interactions with human macrophages. THP-1 macrophages were infected with the wild-type (ISP1820) and its isogenic mutant harbouring a complete deletion of the SPI-2 T3SS (ssaU$s s r B$ ) and intracellular bacteria were determined at different times after infection. Data presented are the mean \pm SEM of three independent experiments performed in duplicate. $P$-values are indicated. similar to that of the wild-type strain (Fig. 3). Despite not demonstrating a key role for intracellular survival, the SPI-2 genes of $S$. Typhi are highly induced inside human macrophages (Faucher et al., 2006), suggesting that the difference may not be at the transcriptional level.

Moreover, no defect in survival was observed for an $s s e B$ SPI-2 mutant of $S$. Typhi strain Ty2, suggesting that the absence of a phenotype for an SPI-2 mutation in S. Typhi was not strain-specific (Fig. 4). However, a phoP mutant of $S$. Typhi known to be involved in intracellular replication of S. Typhimurium (Groisman et al., 1989; Miller et al., 1989) was defective in this model, as was an $s s e B$ mutant of S. Typhimurium (Fig. 4).

As bacterial growth conditions and opsonization affect uptake and have a significant influence on bacterial survival, replication and virulence gene expression in $S$. Typhimurium (Hensel et al., 1997, 1998; Drecktrah et al., 2006), we investigated the influence of growth condition on $S$. Typhi survival in macrophages. Growth to late exponential phase under low aeration promotes SPI-1 expression and T3SS-mediated invasion of $S$. Typhimurium (Lee et al., 1992), while aerated stationary phase bacterial cultures or opsonization promote phagocytosis and low cytotoxicity (Hensel et al., 1998; Lundberg et al., 1999; Drecktrah et al., 2006). Aerated stationary phase or opsonized bacteria are then usually used to study the effect of SPI-2 mutants, as the replication defect of $S$. Typhimurium is overemphasized (Cirillo et al., 1998; Hensel et al., 1998; see also Fig. 5). Under all of these conditions, $S$. Typhi survived and no differences were observed between the wild-type strain and the sseB SPI-2 mutant (Fig. 5). Moreover, there was no significant difference observed in the uptake levels of $S$. Typhi between the different growth conditions or with or without opsonization. The presence of the Vi capsule, specific to $S$. Typhi, may be responsible for the lack of difference in bacterial uptake under different growth conditions. Interestingly, an increase in the number of bacteria recovered $24 \mathrm{~h}$ post-infection (ratio at $24 \mathrm{~h}: 2 \mathrm{~h}$ $>1$ ) was only observed when $S$. Typhi was grown to late exponential phase. It will be interesting to investigate further the mechanisms of entry in macrophages of $S$. Typhi, as they may be related to its survival.

Activated macrophages play a central role in host defence and have been shown to increase phagocytic and killing activities. Survival of $S$. Typhi in human macrophages primed with IFN $-\gamma$ was greatly reduced, but no significant difference was observed between the wild-type and the SPI2 mutant. Similarly, no differences between the wild-type and the SPI-2 mutant were observed during survival in whole human blood (data not shown) or in invasion and survival of human epithelial cells (data not shown). It is possible that SPI-2 effectors may be translocated by the SPI-1 T3SS when the SPI-2 T3SS is mutated, but a double SPI-1 (invA)/SPI-2 (ssaR) mutant in S. Typhi survives and replicates to a level similar to that of the isogenic wild-type strain in THP-1 macrophages (data not shown). Thus, in 
contrast with $S$. Typhimurium, the SPI-2 T3SS of $S$. Typhi is not critical for intracellular survival in human macrophages.

$S$. Typhimurium and $S$. Typhi are closely related serovars, sharing $90 \%$ of their DNA (McClelland et al., 2001). There are no major genomic differences between the SPI-2 T3SS of $S$. Typhimurium and $S$. Typhi that may explain these phenotypic differences (Sabbagh et al., 2010). Phylogenetic analyses suggest a probable difference in functionality between these serovars, as variations in translocon genes sse $C$ and sseD and in effectors sseF and sifA were observed (Eswarappa et al., 2008). The major difference between these serovars seems to be in the diversity of the effector contents, which may contribute to host adaptation. Many SPI-2 effectors characterized in $S$. Typhimurium are missing in S. Typhi (sseI, gogB, spvB, spvC, sseK1, sseK2 and sseK3) or are pseudogenes (sopD2 and sseJ). Several of these effectors mediate long-term systemic infection in mice (Lawley et al., 2006; McLaughlin et al., 2009; RuizAlbert et al., 2002). As some effectors are not present in $S$. Typhi, this emphasizes that there are differences in the virulence mechanisms responsible for systemic disease caused by $S$. Typhimurium in susceptible mice compared with those dictating systemic disease caused by $S$. Typhi in humans. The majority of the studies on maturation and trafficking of $S$. Typhimurium effectors were performed with epithelial cells, and when macrophages were used, they were usually of murine origin. Thus, it is premature to determine or hypothesise whether the absence of a survival defect is related to a difference in the cell type and/or the host source or to a genetic difference between $S$. Typhi and S. Typhimurium.

It is thus possible that the host plays an essential role and may be able to discriminate between these serovars. Indeed, mutations in homologous genes that attenuate $S$. Typhimurium in mice have given different results for $S$. Typhi when administered to humans as a potential vaccine (Garmory et al., 2002). Moreover, differences in survival in human macrophages between the two serovars were previously observed, with a higher rate of survival for $S$. Typhi (Alpuche-Aranda et al., 1994; Ishibashi \& Arai, 1995; Schwan et al., 2000), similar to what we observed here. This may be because $S$. Typhi is less cytotoxic for human cells (Schwan et al., 2000; Fig. 3 and data not shown). S. Typhi increases rapidly and no lag phase is observed in THP-1 macrophages (Figs 2, 3 and 7). Differential survival between these serovars was also detected in susceptible mice, where $S$. Typhi was rapidly eliminated (Xu et al., 2009). The use of $S$. Typhimurium in mice as a surrogate for $S$. Typhi in humans has many real advantages but may also be misleading.

As SPI-2 is present in S. Typhi, it is unlikely that there is no evolutionary advantage in carrying it. It could be used during infection of other cell types, such as dendritic or natural killer cells. The SPI-2 T3SS may be critical when the bacteria are in a specific human niche with high immunological cell content, such as the spleen. The SPI-2 T3SS may be required to modulate the host immune system to establish long-term asymptomatic infection, which will not necessarily translate into defects in intracellular survival. Further investigation is needed to clarify the role of the S. Typhi SPI-2 T3SS in human cells.

\section{ACKNOWLEDGEMENTS}

We are grateful to R. Romieu-Mourez for primary human macrophage preparations and C. Lepage for technical assistance. We thank C. M. Dozois, S. P. Faucher and the members of the Daigle laboratory for critical reading of this manuscript. This research was supported by the Canadian Natural Sciences and Engineering Research Council (NSERC) grant number 251114-06. C.G.F. and S.C.S. were supported by a scholarship from the NSERC.

\section{REFERENCES}

Alpuche-Aranda, C. M., Racoosin, E., Swanson, J. \& Miller, S. (1994). Salmonella stimulate macrophage macropinocytosis and persist within spacious phagosomes. J Exp Med 179, 601-608.

Baron-Bodo, V., Doceur, P., Lefebvre, M., Labroquère, K., Defaye, C., Cambouris, C., Prigent, D., Salcedo, M., Boyer, A. \& Nardin, A. (2005). Anti-tumor properties of human-activated macrophages produced in large scale for clinical application. Immunobiology 210, 267-277.

Basso, H., Rharbaoui, F., Staendner, L. H., Medina, E., Garcia-Del Portillo, F. \& Guzman, C. A. (2002). Characterization of a novel intracellularly activated gene from Salmonella enterica serovar Typhi. Infect Immun 70, 5404-5411.

Beuzón, C. R., Salcedo, S. \& Holden, D. (2002). Growth and killing of a Salmonella enterica serovar Typhimurium sifA mutant strain in the cytosol of different host cell lines. Microbiology 148, 2705-2715.

Brumell, J. H., Rosenberger, C., Gotto, G., Marcus, S. \& Finlay, B. (2001). SifA permits survival and replication of Salmonella typhimurium in murine macrophages. Cell Microbiol 3, 75-84.

Chakravortty, D., Rohde, M., Jäger, L., Deiwick, J. \& Hensel, M. (2005). Formation of a novel surface structure encoded by Salmonella Pathogenicity Island 2. EMBO J 24, 2043-2052.

Chan, K., Kim, C. C. \& Falkow, S. (2005). Microarray-based detection of Salmonella enterica serovar Typhimurium transposon mutants that cannot survive in macrophages and mice. Infect Immun 73, 54385449.

Cheminay, C., Möhlenbrink, A. \& Hensel, M. (2005). Intracellular Salmonella inhibit antigen presentation by dendritic cells. J Immunol 174, 2892-2899.

Cirillo, D. M., Valdivia, R. H., Monack, D. M. \& Falkow, S. (1998). Macrophage-dependent induction of the Salmonella pathogenicity island 2 type III secretion system and its role in intracellular survival. Mol Microbiol 30, 175-188.

Coombes, B. K., Brown, N., Valdez, Y., Brumell, J. \& Finlay, B. (2004). Expression and secretion of Salmonella pathogenicity island-2 virulence genes in response to acidification exhibit differential requirements of a functional type III secretion apparatus and SsaL. J Biol Chem 279, 49804-49815.

Coombes, B. K., Lowden, M., Bishop, J., Wickham, M., Brown, N., Duong, N., Osborne, S., Gal-Mor, O. \& Finlay, B. (2007). SseL is a Salmonella-specific translocated effector integrated into the SsrBcontrolled Salmonella pathogenicity island 2 type III secretion system. Infect Immun 75, 574-580. 
Daigle, F., Graham, J. E. \& Curtiss, R., III (2001). Identification of Salmonella typhi genes expressed within macrophages by selective capture of transcribed sequences (SCOTS). Mol Microbiol 41, 12111222.

Drecktrah, D., Knodler, L. A., Ireland, R. \& Steele-Mortimer, O. (2006). The mechanism of Salmonella entry determines the vacuolar environment and intracellular gene expression. Traffic 7, 39-51.

Eriksson, S., Lucchini, S., Thompson, A., Rhen, M. \& Hinton, J. C. (2003). Unravelling the biology of macrophage infection by gene expression profiling of intracellular Salmonella enterica. Mol Microbiol 47, 103-118.

Eswarappa, S. M., Janice, J., Nagarajan, A. G., Balasundaram, S. V., Karnam, G., Dixit, N. M. \& Chakravortty, D. (2008). Differentially evolved genes of Salmonella pathogenicity islands: insights into the mechanism of host specificity in Salmonella. PLoS ONE 3, e3829.

Faucher, S. P., Porwollik, S., Dozois, C. M., McClelland, M. \& Daigle, F. (2006). Transcriptome of Salmonella enterica serovar Typhi within macrophages revealed through the selective capture of transcribed sequences. Proc Natl Acad Sci U S A 103, 1906-1911.

Faucher, S. P., Forest, C., Béland, M. \& Daigle, F. (2009). A novel PhoP-regulated locus encoding the cytolysin ClyA and the secreted invasin TaiA of Salmonella enterica serovar Typhi is involved in virulence. Microbiology 155, 477-488.

Fields, P. I., Swanson, R., Haidaris, C. \& Heffron, F. (1986). Mutants of Salmonella typhimurium that cannot survive within the macrophage are avirulent. Proc Natl Acad Sci U S A 83, 5189-5193.

Galán, J. E. \& Curtiss, R., III (1989). Cloning and molecular characterization of genes whose products allow Salmonella typhimurium to penetrate tissue culture cells. Proc Natl Acad Sci U S A 86, 6383-6387.

Garmory, H. S., Brown, K. \& Titball, R. (2002). Salmonella vaccines for use in humans: present and future perspectives. FEMS Microbiol Rev 26, 339-353.

Garvis, S. G., Beuzón, C. \& Holden, D. (2001). A role for the PhoP/Q regulon in inhibition of fusion between lysosomes and Salmonellacontaining vacuoles in macrophages. Cell Microbiol 3, 731-744.

Goubau, D., Romieu-Mourez, R., Solis, M., Hernandez, E., Mesplède, T., Lin, R., Leaman, D. \& Hiscott, J. (2009). Transcriptional re-programming of primary macrophages reveals distinct apoptotic and anti-tumoral functions of IRF-3 and IRF-7. Eur J Immunol 39, 527-540.

Groisman, E. A., Chiao, E., Lipps, C. \& Heffron, F. (1989). Salmonella typhimurium phoP virulence gene is a transcriptional regulator. Proc Natl Acad Sci U S A 86, 7077-7081.

Gulig, P. A. \& Curtiss, R., III (1987). Plasmid-associated virulence of Salmonella typhimurium. Infect Immun 55, 2891-2901.

Halici, S., Zenk, S., Jantsch, J. \& Hensel, M. (2008). Functional analysis of the Salmonella pathogenicity island 2-mediated inhibition of antigen presentation in dendritic cells. Infect Immun 76, 4924 4933.

Haraga, A., Ohlson, M. \& Miller, S. (2008). Salmonellae interplay with host cells. Nat Rev Microbiol 6, 53-66.

Helaine, S., Thompson, J., Watson, K., Liu, M., Boyle, C. \& Holden, D. (2010). Dynamics of intracellular bacterial replication at the single cell level. Proc Natl Acad Sci U S A 107, 3746-3751.

Hensel, M., Shea, J., Bäumler, A., Gleeson, C., Blattner, F. \& Holden, D. (1997). Analysis of the boundaries of Salmonella pathogenicity island 2 and the corresponding chromosomal region of Escherichia coli K-12. J Bacteriol 179, 1105-1111.

Hensel, M., Shea, J. E., Waterman, S. R., Mundy, R., Nikolaus, T., Banks, G., Vazquez-Torres, A., Gleeson, C., Fang, F. C. \& Holden, D. W. (1998). Genes encoding putative effector proteins of the type III secretion system of Salmonella pathogenicity island 2 are required for bacterial virulence and proliferation in macrophages. Mol Microbiol 30, 163-174.

Hölzer, S. U. \& Hensel, M. (2010). Functional dissection of translocon proteins of the Salmonella pathogenicity island 2-encoded type III secretion system. BMC Microbiol 10, 104.

Hone, D. M., Harris, A., Chatfield, S., Dougan, G. \& Levine, M. (1991). Construction of genetically defined double aro mutants of Salmonella typhi. Vaccine 9, 810-816.

Ishibashi, Y. \& Arai, T. (1995). Salmonella typhi does not inhibit phagosome-lysosome fusion in human monocyte-derived macrophages. FEMS Immunol Med Microbiol 12, 55-61.

Kaniga, K., Compton, M. S., Curtiss, R., III \& Sundaram, P. (1998). Molecular and functional characterization of Salmonella enterica serovar Typhimurium poxA gene: effect on attenuation of virulence and protection. Infect Immun 66, 5599-5606.

Kuhle, V. \& Hensel, M. (2002). SseF and SseG are translocated effectors of the type III secretion system of Salmonella pathogenicity island 2 that modulate aggregation of endosomal compartments. Cell Microbiol 4, 813-824.

Kuhle, V. \& Hensel, M. (2004). Cellular microbiology of intracellular Salmonella enterica: functions of the type III secretion system encoded by Salmonella pathogenicity island 2. Cell Mol Life Sci 61, 2812-2826.

Lawley, T. D., Chan, K., Thompson, L. J., Kim, C. C., Govoni, G. R. \& Monack, D. M. (2006). Genome-wide screen for Salmonella genes required for long-term systemic infection of the mouse. PLoS Pathog 2, e11.

Lee, C. A., Jones, B. D. \& Falkow, S. (1992). Identification of a Salmonella typhimurium invasion locus by selection for hyperinvasive mutants. Proc Natl Acad Sci U S A 89, 1847-1851.

Leung, K. Y. \& Finlay, B. (1991). Intracellular replication is essential for the virulence of Salmonella typhimurium. Proc Natl Acad Sci U S A 88, 11470-11474.

Lundberg, U., Vinatzer, U., Berdnik, D., von Gabain, A. \& Baccarini, M. (1999). Growth phase-regulated induction of Salmonella-induced macrophage apoptosis correlates with transient expression of SPI-1 genes. J Bacteriol 181, 3433-3437.

McClelland, M., Sanderson, K. E., Spieth, J., Clifton, S. W., Latreille, P., Courtney, L., Porwollik, S., Ali, J., Dante, M. \& other authors (2001). Complete genome sequence of Salmonella enterica serovar Typhimurium LT2. Nature 413, 852-856.

McLaughlin, L. M., Govoni, G., Gerke, C., Gopinath, S., Peng, K., Laidlaw, G., Chien, Y. H., Jeong, H. W., Li, Z. \& other authors (2009). The Salmonella SPI2 effector SseI mediates long-term systemic infection by modulating host cell migration. PLoS Pathog 5, e1000671.

Miller, S. I., Kukral, A. \& Mekalanos, J. (1989). A two-component regulatory system ( $p h o P$ phoQ) controls Salmonella typhimurium virulence. Proc Natl Acad Sci U S A 86, 5054-5058.

Monack, D. M., Detweiler, C. \& Falkow, S. (2001). Salmonella pathogenicity island 2-dependent macrophage death is mediated in part by the host cysteine protease caspase-1. Cell Microbiol 3, 825837.

Muotiala, A. \& Mäkelä, P. (1993). Role of gamma interferon in late stages of murine salmonellosis. Infect Immun 61, 4248-4253.

Nikolaus, T., Deiwick, J., Rappl, C., Freeman, J., Schröder, W., Miller, S. \& Hensel, M. (2001). SseBCD proteins are secreted by the type III secretion system of Salmonella pathogenicity island 2 and function as a translocon. J Bacteriol 183, 6036-6045.

Ochman, H., Soncini, F., Solomon, F. \& Groisman, E. (1996). Identification of a pathogenicity island required for Salmonella survival in host cells. Proc Natl Acad Sci U S A 93, 7800-7804. 
Paesold, G., Guiney, D., Eckmann, L. \& Kagnoff, M. (2002). Genes in the Salmonella pathogenicity island 2 and the Salmonella virulence plasmid are essential for Salmonella-induced apoptosis in intestinal epithelial cells. Cell Microbiol 4, 771-781.

Parkhill, J., Dougan, G., James, K., Thomson, N. R., Pickard, D., Wain, J., Churcher, C., Mungall, K. L., Bentley, S. D. \& other authors (2001). Complete genome sequence of a multiple drug resistant Salmonella enterica serovar Typhi CT18. Nature 413, 848-852.

Pfeifer, C. G., Marcus, S., Steele-Mortimer, O., Knodler, L. \& Finlay, B. (1999). Salmonella typhimurium virulence genes are induced upon bacterial invasion into phagocytic and nonphagocytic cells. Infect Immun 67, 5690-5698.

Romieu-Mourez, R., Solis, M., Nardin, A., Goubau, D., Baron-Bodo, V., Lin, R., Massie, B., Salcedo, M. \& Hiscott, J. (2006). Distinct roles for IFN regulatory factor (IRF)-3 and IRF-7 in the activation of antitumor properties of human macrophages. Cancer Res 66, 1057610585.

Rosenberg, S. A., Spiess, P. \& Kleiner, D. (2002). Antitumor effects in mice of the intravenous injection of attenuated Salmonella typhimurium. J Immunother 25, 218-225.

Ruiz-Albert, J., Yu, X., Beuzón, C., Blakey, A., Galyov, E. \& Holden, D. (2002). Complementary activities of SseJ and SifA regulate dynamics of the Salmonella typhimurium vacuolar membrane. Mol Microbiol 44, 645-661.

Sabbagh, S. C., Forest, C. G., Lepage, C., Leclerc, J. M. \& Daigle, F. (2010). So similar, yet so different: uncovering distinctive features in the genomes of Salmonella enterica serovars Typhimurium and Typhi. FEMS Microbiol Lett 305, 1-13.

Santiviago, C. A., Reynolds, M., Porwollik, S., Choi, S., Long, F., Andrews-Polymenis, H. \& McClelland, M. (2009). Analysis of pools of targeted Salmonella deletion mutants identifies novel genes affecting fitness during competitive infection in mice. PLoS Pathog 5, e1000477.

Schwan, W. R., Huang, X. Z., Hu, L. \& Kopecko, D. J. (2000). Differential bacterial survival, replication, and apoptosis-inducing ability of Salmonella serovars within human and murine macrophages. Infect Immun 68, 1005-1013.
Segura, I., Casadesús, J. \& Ramos-Morales, F. (2004). Use of mixed infections to study cell invasion and intracellular proliferation of Salmonella enterica in eukaryotic cell cultures. J Microbiol Methods 56, 83-91.

Shea, J. E., Hensel, M., Gleeson, C. \& Holden, D. W. (1996). Identification of a virulence locus encoding a second type III secretion system in Salmonella typhimurium. Proc Natl Acad Sci U S A 93, 2593-2597.

Shea, J. E., Beuzon, C., Gleeson, C., Mundy, R. \& Holden, D. (1999). Influence of the Salmonella typhimurium pathogenicity island 2 type III secretion system on bacterial growth in the mouse. Infect Immun 67, 213-219.

Srinivasan, J., Tinge, S., Wright, R., Herr, J. \& Curtiss, R., III (1995). Oral immunization with attenuated Salmonella expressing human sperm antigen induces antibodies in serum and the reproductive tract. Biol Reprod 53, 462-471.

Tobar, J. A., Carreño, L., Bueno, S., González, P., Mora, J., Quezada, S. \& Kalergis, A. (2006). Virulent Salmonella enterica serovar Typhimurium evades adaptive immunity by preventing dendritic cells from activating T cells. Infect Immun 74, 6438-6448.

Uchiya, K., Groisman, E. \& Nikai, T. (2004). Involvement of Salmonella pathogenicity island 2 in the up-regulation of interleukin-10 expression in macrophages: role of protein kinase A signal pathway. Infect Immun 72, 1964-1973.

Valdivia, R. H. \& Falkow, S. (1997). Fluorescence-based isolation of bacterial genes expressed within host cells. Science 277, 2007-2011.

Waterman, S. R. \& Holden, D. (2003). Functions and effectors of the Salmonella pathogenicity island 2 type III secretion system. Cell Microbiol 5, 501-511.

Xu, T., Maloy, S. \& McGuire, K. (2009). Macrophages influence Salmonella host-specificity in vivo. Microb Pathog 47, 212-222.

Yu, X. J., Liu, M. \& Holden, D. (2004). SsaM and SpiC interact and regulate secretion of Salmonella pathogenicity island 2 type III secretion system effectors and translocators. Mol Microbiol 54, 604619.

Edited by: P. H. Everest 